\title{
EVALUACIÓN DEL TRABAJO DE GRUPO A TRAVÉS DE LA PARTICIPACIÓN EN PROYECTOS DE APRENDIZAJE TUTORADO. REFLEXIONES Y PROPUESTAS
}

Assessment of group work through participation in Project Oriented Learning.

Reflections and proposals

Avaliação do grupo de trabalho através da participação de um Projeto de Aprendizagem Explicações Co. Reflexões e propostas

Miriam Sonlleva Velasco (1)

Suyapa Martínez Scott (2)

\section{Roberto Monjas Aguado (3)}

(1) Universidad de Valladolid. Teléfono: +34 9211122 94. miriam.sonlleva@uva.es.

(2) Universidad de Valladolid. Teléfono: +34 9211122 94. suyapa@pdg.uva.es.

(3) Universidad de Valladolid. Teléfono: +31 9211123 21. rmonjas@gmail.com

\section{Resumen}

Presentamos la puesta en práctica de un Proyecto de Aprendizaje Tutorado Cooperativo (PAT), a través del Puzzle de Aronson y coordinado entre dos de las asignaturas del Título de Grado de Educación Infantil: Educación para la paz y la igualdad y Organización y planificación escolar. Materias que se imparten en el primer año de carrera, con carácter básico y con una carga lectiva de 6 créditos ECTS. Entre los objetivos propuestos destacamos: eliminar el efecto polizón (Slavin, 1999) y conseguir la participación equilibrada de los integrantes del grupo de trabajo, así como mejorar los aprendizajes obtenidos y buscar alternativas para evaluar y calificar los trabajos grupales en el ámbito universitario. La evaluación que se ha llevado a cabo en los PAT nos lleva a que asignar responsabilidades individuales dentro de los PAT, así como llevarlos a la práctica en un contexto real próximo a su futura labor como docentes, parecen dos medidas importantes para que los estudiantes asuman un mayor compromiso con el trabajo grupal.

Palabras clave: Formación inicial del profesorado; Educación Infantil; Proyecto de Aprendizaje Tutorado Cooperativo; Evaluación Formativa

Evaluación del trabajo de grupo a través de la participación en proyectos de aprendizaje tutorado. Reflexiones y propuestas 


\begin{abstract}
We present a proposal for the implementation of a Cooperative Project Oriented Learning (POL), through the Aronson Puzzle. The proposal is coordinated between two of the subjects of the Degree of Early Childhood Education: Education for peace and equality and Organization and planning school. These two subjects are taught in the first year of the Degree and have got a basic character and 6 ECTS credits. Among the proposed objectives, we highlight the elimination of the stowaway effect (Slavin, 1999) and the balanced participation of the members of the work groups, as well as to improve the obtained learning and to find alternatives to qualify the group work in the university area. The assessment carried out in the PATs leads us to think that granting individual responsibilities within the PAT, as well as putting them into practice in a real context close to their future work as teachers, seem two important measures for students to get a greater commitment to group work.
\end{abstract}

Keywords: Preservice teacher training; Early childhood Education; Cooperative Project Oriented Learning; Formative Assessment

\title{
Resumo
}

Nós apresentamos uma proposta de implementação de um Projeto de Aprendizagem Explicações Co (PAT), através do quebra-cabeça Aronson e coordenado entre dois dos assuntos da Licenciatura em Educação Infantil: Educação para a paz e igualdade, e Organização e planejamento escolar. Matérias ensinadas no primeiro ano de corridas, com básicos e 6 ECTS. Entre os objetivos propostos incluem: para eliminar o efeito clandestino (Slavin, 1999) e alcançar uma participação equilibrada dos membros do grupo de trabalho e melhorar a aprendizagem obtida e buscar alternativas para qualificar o trabalho de grupo em nível universitário. A avaliação foi realizada no PAT nos leva a crer que a concessão de responsabilidades individuais dentro do PAT e implementá-las no contexto perto real para seu futuro trabalho como professores, eles parecem duas medidas importantes para estudantes assumir um maior compromisso com o trabalho em grupo.

Palavras-chave: Formação inicial de professores; Educação infantil; Explicações Projeto de Aprendizagem Cooperativa; avaliação formativa

Evaluación del trabajo de grupo a través de la participación en proyectos de aprendizaje tutorado. Reflexiones y propuestas 


\section{Introducción}

El Proyecto de Aprendizaje Tutorado (PAT) es una forma de trabajo grupal relacionada con el futuro profesional de los maestros en formación, tutorizado por el profesorado y que se desarrolla en pequeños grupos autónomos (Meyer, 2002). Esta forma de trabajo favorece el aprendizaje deliberativo (Bronkhorst, Meijer, Koster, y Vermunt, 2011), que permite aprender a partir del diálogo igualitario, la generación de ideas y el desarrollo de habilidades (Gentili et al., 1997).

Una limitación del trabajo grupal es la implicación personal de cada uno de sus componentes. Evaluar estos trabajos suele ser algo complicado, pues conlleva conocer cuál es el nivel de conocimiento logrado por cada estudiante. Por ello, vinculamos el PAT con la evaluación formativa siguiendo la línea de Barba, López, Gea, y Monjas (2010); López (2008); y Martínez, Gea y Barba (2014).

\section{Contextualización}

El trabajo mediante el PAT es una metodología empleada en el ámbito universitario e implementada en nuestro centro desde hace más de diez años (Barba, Martínez y Torrego, 2012; Barba et al., 2010; López et al., 2010; Manrique, López, Monjas y Real, 2010; Martínez et al., 2014).

La experiencia que presentamos se ha llevado a cabo con un grupo de 43 estudiantes del primer curso de Grado en Educación Infantil (EI), en dos asignaturas básicas de la titulación: Organización y planificación escolar y Educación para la paz y la igualdad (6 créditos ECTS cada una). En ellas se lleva a cabo un PAT que sirve como parte de la evaluación y calificación de ambas asignaturas. Este PAT se enmarca dentro del Proyecto de Innovación Docente de la UVa: TitiriUVa (Pérez, Gómez, Gil, et. al, 2016). Unimos en un solo trabajo una propuesta con amplia tradición en nuestro campus, el PAT, con un proyecto fuertemente arraigado en nuestra ciudad: Titirimundi ${ }^{1}$, mediante una metodología cooperativa y una evaluación formativa y compartida, todo ello en colaboración con varios centros educativos de Segovia.

\section{Diseño y desarrollo}

Los objetivos propuestos al emplear el PAT basado en el aprendizaje cooperativo son:

\footnotetext{
${ }^{1}$ Titirimundi: Festival Internacional de Teatro de Títeres celebrado en Segovia desde 1985 y con proyección a nivel mundial (Cebrián, 2016).

Evaluación del trabajo de grupo a través de la participación en proyectos de aprendizaje tutorado. Reflexiones y propuestas 
- Favorecer el desarrollo y evaluación de los trabajos grupales con una participación equilibrada que elimine el efecto polizón (Salvin, 1999).

- Evaluar la puesta en práctica de los PAT, exponiendo reflexiones y propuestas que contribuyan a mejorar la interacción y el aprendizaje que desarrollan los estudiantes universitarios.

- Analizar y valorar si este tipo de trabajo contribuye a evitar la desconexión entre las Facultades de Educación y los centros escolares.

Este PAT se basa en la estructura del puzzle de Aronson, adaptada como muestra la Tabla 1. La evaluación de este trabajo está relacionada con la calificación. Esto se refleja en ambas asignaturas con un porcentaje del 40\% (Tabla 1).

Tabla 1.

El puzzle de Aronson en el PAT de EI

\begin{tabular}{|c|c|c|}
\hline $\begin{array}{l}\text { Fases del } \\
\text { PAT }\end{array}$ & Actuaciones alumnos & Actuaciones docentes \\
\hline $\begin{array}{l}\text { FASE A: } \\
\text { Formación } \\
\text { expertas }\end{array}$ & $\begin{array}{l}\text { En cada grupo existen cinco roles que exigen } \\
\text { que cada persona se convierta en experta en } \\
\text { un tema clave del PAT. Al finalizar, elabora } \\
\text { un informe individual que se comparte con el } \\
\text { grupo. En él se recoge información relevante } \\
\text { para la siguiente fase. }\end{array}$ & $\begin{array}{l}\text { Orienta, tutoriza y evalúa el informe. } \\
\text { Una vez evaluado se devuelve a cada } \\
\text { estudiante para que pueda mejorarlo } \\
\text { en el plazo de una semana. } \\
\text { Calificación individual que supone un } \\
\text { 10\% de la nota del PAT. }\end{array}$ \\
\hline $\begin{array}{l}\text { FASE B: } \\
\text { Programación } \\
\text { talleres }\end{array}$ & $\begin{array}{l}\text { Programación del taller de títeres que se lleva } \\
\text { a cabo con alumnado de EI en la Universidad } \\
\text { ( } 20 \text { min). Programación del taller de una hora } \\
\text { que se lleva a cabo en un aula de EI. Estas } \\
\text { propuestas trabajan uno de los bloques de la } \\
\text { asignatura de Educación para la paz y la } \\
\text { igualdad. }\end{array}$ & $\begin{array}{l}\text { Coordinación con centros educativos } \\
\text { y compañeros de la universidad. } \\
\text { Orientación, tutorización y guía a los } \\
\text { grupos. Evalúa el trabajo } \\
\text { periódicamente empleando rúbricas } \\
\text { que los estudiantes conocen de } \\
\text { antemano. Calificación grupal que } \\
\text { supone un 20\% de la nota del PAT. }\end{array}$ \\
\hline $\begin{array}{l}\text { FASE C: } \\
\text { Puesta en } \\
\text { práctica }\end{array}$ & $\begin{array}{l}\text { Realización del taller de títeres con alumnado } \\
\text { de EI y puesta en práctica del PAT en un aula. } \\
\text { Observación de otros grupos para coevaluar } \\
\text { (ficha de observación proporcionada por los } \\
\text { docentes). }\end{array}$ & $\begin{array}{l}\text { Coordinación con centros educativos } \\
\text { y compañeros de la universidad. } \\
\text { Orientación, tutorización y guía a los } \\
\text { grupos. Evalúa la puesta en práctica } \\
\text { antes de ir a los centros. } \\
\text { (Calificación }=10 \%) \text {. }\end{array}$ \\
\hline $\begin{array}{l}\text { FASE D: } \\
\text { Elaboración } \\
\text { definitiva }\end{array}$ & $\begin{array}{l}\text { A la programación del taller hay que añadir la } \\
\text { continuidad del trabajo en ese componente de } \\
\text { Educación para la paz y la igualdad a lo largo } \\
\text { del tiempo. En esta fase se realizan cambios } \\
\text { en la programación del taller en función de la } \\
\text { experiencia vivida y se incluye la evaluación } \\
\text { del taller. }\end{array}$ & $\begin{array}{l}\text { Orientación, guía, tutorización y } \\
\text { evaluación de los trabajos. La } \\
\text { calificación de esta parte es la misma } \\
\text { que la de la Fase B }\end{array}$ \\
\hline $\begin{array}{l}\text { FASE E: } \\
\text { Evaluación } \\
\text { del PAT }\end{array}$ & $\begin{array}{l}\text { Realización del informe de evaluación } \\
\text { individual. } \\
\text { Sesión de evaluación final donde cada grupo } \\
\text { expone su trabajo al resto. Estudiantes y } \\
\text { docentes valoran el desarrollo del PAT. }\end{array}$ & $\begin{array}{l}\text { Informe de evaluación final donde se } \\
\text { valora el aprendizaje obtenido. } \\
\text { Dinamiza la sesión de evaluación } \\
\text { grupal. } \\
\text { Califica el PAT. }\end{array}$ \\
\hline
\end{tabular}

Fuente: Elaboración propia

Evaluación del trabajo de grupo a través de la participación en proyectos de aprendizaje tutorado. 


\section{Evaluación y conclusiones}

Llevamos tres cursos trabajando desde la propuesta que presentamos, lo que nos ha permitido elaborar una serie de conclusiones a partir, fundamentalmente, de los informes de evaluación que elabora el alumnado, de las sesiones finales de evaluación conjunta y del análisis y revisión conjunta del equipo de docentes que participan en el proyecto. En relación a los objetivos planteados encontramos que:

- Unir el aprendizaje cooperativo con los PAT promueve la eliminación del efecto polizón (Slavin, 1999), ya que se consigue la participación de todos en la elaboración del PAT, gracias al Puzzle de Aronson.

- Otorgar responsabilidades individuales es una medida más eficaz que otras estructuras organizativas en las que se comparte la calificación y los estudiantes optan por eludir responsabilidades.

- La supervisión del docente durante el proceso hace que los estudiantes se sientan con más responsabilidad al tener que entablar diálogos sobre la temática del trabajo y su evaluación.

- La puesta en práctica del PAT con escolares, aumenta las exigencias de los estudiantes en cuanto a la responsabilidad aún mayor que si el trabajo fuera realizado únicamente para ser entregado.

- La coordinación interdisciplinar, pese a suponer un mayor esfuerzo, es un punto que los estudiantes valoran muy positivamente.

- Este trabajo puede contribuir a proporcionar alternativas para una cuestión compleja como el desarrollo de trabajos grupales en la docencia universitaria y mostrar otras vías para su evaluación y calificación.

\section{Referencias}

Barba Martín, J. J., López Pastor, V.M, Manrique Arribas, J.C., Gea Fernández, J.M., \& Monjas Aguado, R. (2010). Garantir l'èxit en la formació inicial del professorat d'educació física: Els projectes d'aprenentatge tutelats. Temps d'Educacio, 39, 187-206.

Evaluación del trabajo de grupo a través de la participación en proyectos de aprendizaje tutorado. 
Barba Martín, J. J., Martínez Scott, S., \& Gea Fernández, J.M. (2014). Project Oriented Learning: the use of formative evaluation for the improvement of the group work. Revista Pedagogica Adal 28, 15-20. Retrieved from: https://issuu.com/apefadal/docs/revista_28_final

Barba Martín, J. J., Martínez Scott, S., \& Torrego Egido, L. (2012). El proyecto de aprendizaje tutorado cooperativo: Una experiencia en el grado de maestra de educación infantil. REDU. Revista de Docencia Universitaria, 10(1), 123-144. Retrieved from: http://redaberta.usc.es/redu/index.php/REDU/article/view/310/pdf

Bronkhorst, L. H., Meijer, P. C., Koster, B., \& Vermunt, J. D. (2011). Fostering meaning-oriented learning and deliberate practice in teacher education. Teaching and Teacher Education, 27(7), 1120-1130. doi:10.1016/j.tate.2011.05.008

Cebrián Velasco, B. (2016). El títere y su valor educativo: análisis de su influencia en Titirimundi: Festival Internacional de Títeres de Segovia. (Tesis doctoral). Retrieved from: http://uvadoc.uva.es/bitstream/10324/16249/1/Tesis838160224.pdf

Gentili, K., Hannan,J., Crain, R., Davis, D., Trevisan, M., \& Calkins, D. (1997). A process oriented class in engineering design: How it works. Frontiers in Education 1997 - 27 $7^{\text {th }}$ Annual Conference, Proceedings, Vols I - III, 972 - 980. Retrieved form: http://fie-conference.org/fie97/papers/1129.pdf

López Pastor, V.M. (2008). Implementing a formative and shared assesment system in higher education teaching. European Journal of Teaching Education, 31 (3), 293 $-311$.

López Pastor, V.M., Monjas Aguado, R., \& Gea Fernández, J.M. (2010). Formative assessment in projet oriented learning to improve academmic performance. Assessment, Teaching Learning Journal, 9, 23-26.

Manrique Arribas, J.M., López Pastor, V.M., Monjas Aguado, R. y Real Rubio, F. (2010). El potencial de los Proyectos de Aprendizaje Tutorado y los sistemas de evaluación formativa en la mejora de la autonomía del alumnado. Una experiencia 
interdisciplinar en formación inicial del profesorado. REEFD, 388 (14) Retrieved from: http://www.reefd.es/index.php/reefd/article/view/285/276

Meyer, V. (2002). Project oriented learning (POL) as a communication tool of environmental sciences in the community of sohanguve. A case study. Paper presented at the International Conference on Sustainability of Water Resources, Murdoch University, Western Australia. Retrieved from: www.saasta.ac.za/scicom/pcst7/meyer_v.pdf

Pérez Brunicardi, D., Gómez Redondo, C., Gil Puente, C., Archilla Prat, M., López Luengo, M.A, Cienfuegos Antelo, G., Vallés Rapp, C., Monjas Aguado, R., Maroto Sáez, A., Martínez Scott, S. (2016). TITIRIUVA. Un proyecto internivelar e interdisciplinar de formación de estudiantes de la Facultad de Educación de Segovia a través de los títeres. Universidad de Valladolid. Área de Formación Permanente e Innovación Docente. Retrieved from: http://uvadoc.uva.es/bitstream/10324/18426/1/TITIRIUVA-Jornada-

\section{Innovaci\%C3\%B3n-Docente.pdf}

Slavin, R.E. (1999). Aprendizaje cooperativo. Teoría, investigación y práctica. Argentina: Aique. 